\title{
A FUZZY DNA-BASED ALGORITHM FOR IDENTIFICATION AND AUTHENTICATION IN AN IRIS DETECTION SYSTEM
}

\author{
Alberto de Santos Sierra \\ Centro de Domótica Integral \\ Universidad Politécnica de Madrid \\ Madrid, 28223 \\ Spain \\ alberto@cedint.upm.es
}

\author{
Carmen Sánchez Ávila \\ Centro de Domótica Integral \\ Universidad Politécnica de Madrid \\ Madrid, 28223 \\ Spain \\ csa@mat.upm.es
}

\author{
Vicente Jara Vera \\ Applied Math. Department \\ ETSI de Telecomunicación \\ Madrid, 28040 \\ Spain \\ vjara@mat.upm.es
}

\begin{abstract}
Several algorithms have been proposed in order to identify or authenticate a certain user in an Iris Detection System. These previous algorithms are based on different transforms applied to a certain features extracted from the Iris, known as Iris template. However, this new approach aims to identify and/or authenticate a user avoiding transforms, comparing directly the Iris templates instead. Based on ideas inherited from DNA alignment algorithms and fuzzy logic, this new algorithm attains a better result in deciding whether two templates belong to the same user, when being compared to previous algorithms. Furthermore, this new algorithm allows a partial comparison between the whole template stored in a database, and a portion of template, decreasing the accessing time. As a result, not only False Acceptance Rate (FAR) and False Rejection Rate (FRR) but also time in identifying and authenticating are highly decreased compared to current algorithms and furthermore its flexibility in a local comparison makes of it a promising algorithm in not only an Iris Detection System, but in any Biometric System.
\end{abstract}

Index Terms-Authentication, Biometry, DNA Global and Local Alignment, Fuzzy Logic, Identification, Iris Detection System, Iris Template.

\section{INTRODUCTION}

Attempting to recognize an individual based on the Iris covers several steps since the image is acquired till the system is able to accept or reject the previous mentioned individual. Firstly, the information contained in the irides must be extracted. Several patterns and templates has been proposed, together with algorithms able to compare in a precise manner the former features and elucidate whether or not two patterns are the same, [1], [2], [3].

On the other hand, there exist algorithms able to compare DNA sequences, containing proteins or aminoacids. These algorithms provide an alignment among sequences, allowing to know whether two sequences remain the same, or on the contrary, mutation has occurred along evolution. In other words, DNA alignment algorithm gives an idea about how similar two protein sequences are, [4].

The purpose of this paper consists of gathering the former ideas, id est, implement an algorithm able to align two Iris templates. However, DNA alignment algorithms must be tuned in order to make possible such an alignment between two templates. Fuzzy logic will provide this new algorithm with the required skills to meet the final goals.

Furthermore, this algorithm has been tested in UPOL database, [5], with very promising results.

Once the problem has been stated, the papers goes on with a detailed explanation of not only the algorithm itself but also about how the information is extracted from the Iris. Furthermore, results are presented and compared, ending with conclusions and future work ${ }^{1}$.

\section{ALIGNING TWO IRIS TEMPLATES}

Within this section, several aspects are gathered. First of all, how the information is extracted from the Iris is explained. Once the features have been extracted, how to decide whether two individuals are the same is described. Background in DNA Alignment is also given for the sake of understanding. Finally, this section ends presenting the results obtained and a comparison with previous works based on the same database.

\section{A. Feature Extraction}

Since a biometric system relies strongly on how the information is extracted from the relevant biometric technique, feature extraction deserves being treated separately.

Although several feature extraction schemes have been already proposed, [1], [2], [6], [7], [8], this paper will focus on those templates suggested by Boles et al., [9], and Sánchez-Ávila et al., [10]. Inspired by these two previous schemes, the pattern provided within this paper comes up.

First of all, a segmentation process is carried out in order to ensure the fact that only Iris tissue is extracted in subsequent steps, id est, Iris is isolated from a given eye image, neglecting not only eyelashes but also eyelids. Furthermore, pupil radius and center are extracted.

Straight afterwards, and taking pupil radius and center from previous segmentation as an input parameter, a virtual

\footnotetext{
${ }^{1}$ The authors would like to thank Proyecto CENIT Segur@: Seguridad y Confianza en la Sociedad de la Información, financed by Ministerio de Industria, Turismo y Comercio.
} 
crown of radius $\rho$ is extracted from the Iris as follows: Considering the fact that the crown can be divided into 360 degrees, for a given angle $\alpha \in \mathbb{N} \cap[1,360]$, intensities along radius $\rho$ are recorded and averaged. In other words, the template consists of 360 points, result of the previous radial average of intensities.

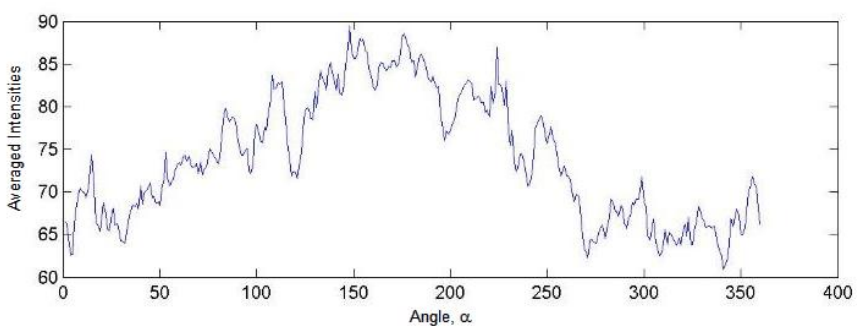

Fig. 1. Feature extracted from an Iris image

As an example, Fig. 1 provides a visual performance of the extraction of features in a random image in UPOL database, [5], where reader can notice the 1-D nature of this feature.

Once the feature has been extracted, a desired goal of any Iris biometric system is to tackle the challenge of deciding whether two irides are the same or not, based on previous feature.

\section{B. Algorithm Specification}

Measuring similarities between two patterns is a very well-known problem in pattern recognition, [11]. Within this paper, a new approach based on DNA Alignment algorithms is proposed to cope with the problem of comparing two onedimensional signals.

Furthermore, and due to the nature of the DNA Alignment algorithms, new possibilities emerge such as local alignments, id est, recognize part of the template from a whole template, allowing, exempli gratia, to identify an individual even from a partial occluded eye, or storing a big template in the system, requiring only a small template to access inside, reducing accessing time. Besides, this technique can be used to avoid a $1: N$ comparison when seeking for the identity of the accessing user in a database due to a properties of the algorithms themselves.

Apart from this, global alignment might be also performed, keeping all the properties of a suitable comparison algorithm.

This two aspects of the algorithm will be specifically described in subsequent subsections.

1) Global Alignment: The first alignment algorithm was designed by Needlmand and Wunsch, [12], around 1970. Based on Dynamic Programming, the algorithm was able to find an optimal alignment according to a certain matrix (substitution matrix) where the score of aligning two aminoacids (or proteins) was established. This algorithm considered the option of adding 'gaps' between sequences in order to emphasize the idea of mutation (by substitution, deletion or insertion of new aminoacids), and these gaps were included also in the original previous substitution matrix. An example of global alignments with aminoacid sequences is provided in Fig. 2, where reader can realize visually of both the concept of alignment and gap.
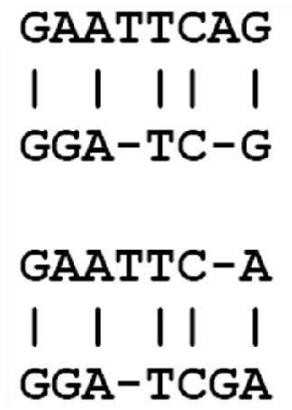
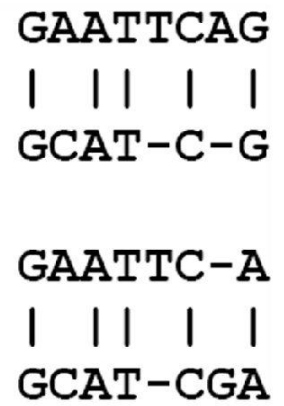

Fig. 2. Several global alignments for aminoacid sequences

However, in an attempt to adapt the original idea of Needleman and Wunsch, [4], [12], there exist several aspects which require a new approach.

First aspect regards 'gaps', in other words, the space included when a mutation occurs. Gaps are represented in the original algorithm by a hyphen (-), but within this new algorithm neither the representation nor the concept is longer available. Since gaps measure how two sequences have changed along time (mutation), gaps might be then a good measurement of how different two sequences are. In other words, gaps will represent, in this new approach, the form of deciding whether two templates are the same or not. The higher the number of gaps, the lower the similarity.

On the other hand, representation remains to be solved. Gaps contains a peculiarity: 'It must not be included within the alphabet', being this the reason why gaps are represented by a hyphen. Notice that in original algorithm, sequences are represented by letters $(A, C, G, T$ in case of aminoacids, F, Y, W, C, M, V, L, I, H, K, R, E, D, Q, N, P, A, G, S, T in case of proteins, [4]), so gaps can be represented by hyphen, since they are not included within the alphabet.

Therefore, gaps must be represented by a number whose values might never be included in the values of the templates. Then, considering the fact that templates contains average values of gray colors, black (represented by 0 ) will never be included on them, for there exist none whose Iris (or at least one region of it) is black.

Thus, gaps are represented by zeros, 0 , and they will provide an idea of how different two templates are.

Second aspect regards substitution matrix. Originally, there exist several matrix which gather all the possible alignments among proteins or aminoacids, exempli gratia, 
BLOSUM62, PAM250, [4], among others. However, since alphabet in original algorithm is delimited (four elements in case of aminoacids, twenty in case of proteins), such a substitution matrix was a suitable solution. Nevertheless in this new approach, due to the nature of the templates. Reader must notice that templates contain elements which belong to $\mathbb{R}$ since they are average of integer values, $\mathbb{N}$.

In order to tackle this problem, fuzzy logic provides a very suitable solution. Since substitution matrix comes out with a measurement of an alignment between two elements, a function able to measure such a similarity should verify to be symmetrical and congruent with gaps representation, besides of giving a measurement of how similar two values in different templates are. Instead of a discrete substitution matrix, a gaussian function will verify previous conditions.

Two parameters are required to define a gaussian normal distribution, [13]: $\sigma$, representing standard deviation, and $\mu$, mean. This function, as it can be seen in Fig. 3 , is symmetrical with regard to mean $\mu$. Furthermore, if two values are to be compared, one will be considered as the mean $\mu$, giving the function itself a measure of how this latter value differs from the former one. Finally, this gaussian function is congruent with the chosen representation of the gap, since aligning each value with 0 will result in a low score.

Mathematically, this function is represented by $\zeta$, and let be $T_{1}$ and $T_{2}$ two templates belonging to the set of all possible templates $\mathcal{T}$, then $T_{1}^{i}$ corresponds to the value $i^{\text {th }}$ of template $T_{1}$ and $T_{2}^{j}$ corresponds to the value $j^{\text {th }}$ of template $T_{2}$, with $i, j \in\{1, \ldots, 360\}$. Therefore, $\zeta\left(T_{1}^{i}, T_{2}^{j}\right) \in(0,1]$ gives a measurement of the similarity between $T_{1}^{i}$ and $T_{2}^{j}$. Reader might notice that $\zeta$ verify the property of commutativity, id est, $\zeta\left(T_{1}^{i}, T_{2}^{j}\right)=\zeta\left(T_{2}^{j}, T_{1}^{i}\right)$.

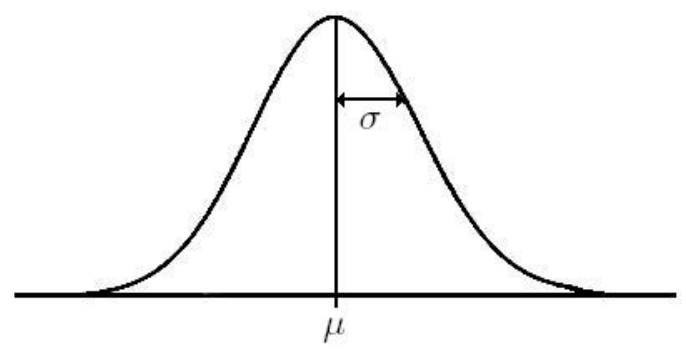

Fig. 3. Fuzzy distribution for substitution matrix

Range for $\zeta$ is $(0,1]$ due to the fact that theoretically $\zeta$ could never be null. Instead, and concerning computational errors, $\zeta$ can be null, without any significance for this algorithm. In Fig. 4, a fuzzy substitution matrix, $\sigma=50$, can be seen.

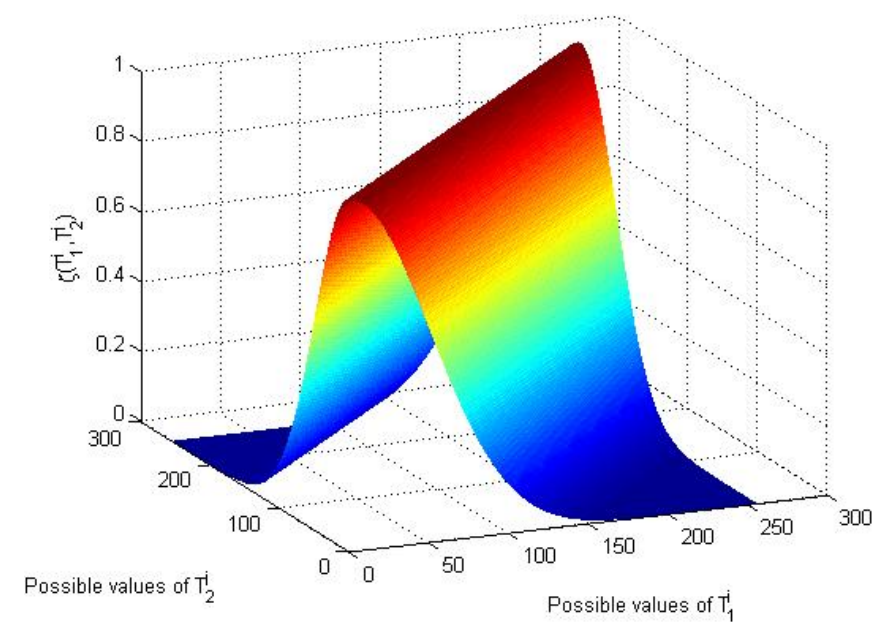

Fig. 4. A fuzzy substitution matrix

Evidently, $\sigma$ is a very important parameter since it can manage to what extend two elements $T_{1}^{i}$ and $T_{2}^{j}$ are similar or not. In section II-C, a study concerning $\sigma$ is carried out concluding that an optimal $\sigma$ can be found to minimize Equal Error Rate, EER, and thus, maximize the performance of the whole system.

Finally, the algorithm based on Dynamic Programming must be adapted to the characteristics of the problem. However, this modification is negligible compared to previous changes, id est, gaps and substitution matrix. Firstly, the problem must be formally stated: Given two templates $T_{1}, T_{2} \in \mathcal{T}, M$ is the best alignment regarding gaps metrics (the greater the number of gaps, the lower the similarity), and function $\zeta$. This is actually a very difficult problem, but easily solved by Dynamic Programming as follows:

$$
M[i, j]=\max \left\{\begin{array}{l}
M[i-1, j-1]+\zeta\left(T_{1}^{i}, T_{2}^{j}\right) \\
M[i-1, j]-\zeta\left(T_{1}^{i}, 0\right) \\
M[i, j-1]-\zeta\left(T_{2}^{j}, 0\right)
\end{array}\right.
$$

with $M[1,1]=0$ as a common criterion, [4].

A backtracing algorithm is carried out to find out the optimal path through $M$. In order to understand how this backtracing algorithm works, a deeper explanation on previous equation must be provided. Let focus on position $M[i, j]$. In case maximum value is $M[i-1, j-1]+\zeta\left(T_{1}^{i}, T_{2}^{j}\right)$, then $T_{1}^{i}$ and $T_{2}^{j}$ can be aligned together. This is called substitution. In case maximum value is $M[i-1, j]-\zeta\left(T_{1}^{i}, 0\right)$, then $T_{1}^{i}$ is aligned with the gap. This is called insertion. Eventually, in case maximum value is $M[i, j-1]-\zeta\left(T_{2}^{j}, 0\right)$, then $T_{2}^{j}$ is aligned with the gap. This is called deletion.

This short explanation is compulsory to understand what a backtracing algorithm makes in a global alignment. Beginning from $M[360,360]$, this backtrace procedure checks 
which of the surrounding cells in matrix $M, M[360,360]$ comes from. This is made iteratively, till $M[1,1]$ is achieved.

As a result of this algorithm two new sequences are obtained, $\widetilde{T}_{1}$ and $\widetilde{T}_{2}$, which contain the original templates together with the included gaps.

There exists another possibility between global and local alignment known as semi-global alignment, which takes as a first value to begin backtracing algorithm, not $M[360,360]$, but the maximum value along the sides of matrix $M$, id est, column $j$ and row $i$. However, this alignment lacks of interest within this context.

2) Local Alignment: As it was introduced before, local alignment would allow certain improvements. First of all, the problem must be stated formally: Let be $T, U, V \in \mathcal{T}$, where $T$ and $U$ belong to the same user, and $V$ to another, and let be $\tau$ a partial section of previous $T$. Problem to be solved is whether $\tau$ belongs to $U$, and what is more, to what extend $\tau$ belongs to $V$.

This problem can be easily solved by means of Dynamic Programming again, based on Smith-Waterman algorithm, [14]. This algorithm presents two differences in relation to previous global alignment: Firstly, in finding $M$ there exists an extra possibility for each cell, and secondly, maximum value in the whole matrix $M$ is considered, instead of $M[360,360]$.

Since there is another possibility, algorithm to find $M$ changes as follows:

$$
M[i, j]=\max \left\{\begin{array}{l}
0 \\
M[i-1, j-1]+\zeta\left(T_{1}^{i}, T_{2}^{j}\right) \\
M[i-1, j]-\zeta\left(T_{1}^{i}, 0\right) \\
M[i, j-1]-\zeta\left(T_{2}^{j}, 0\right)
\end{array}\right.
$$

with $M[1,1]=0$ as a common criterion, [4], as before. Taking the option 0 corresponds to starting a new alignment. In other words, if the best alignment up to some point has a negative score, it is a better option to start a new one, rather than extend the old one.

Actually, this local alignment is used to find small protein chains within a long protein sequence, as it can be seen in Fig. 5.

\section{CAGCA-CTTGGATTCTCGG ---CAGCGTGG--------}

Fig. 5. Local alignment example with aminoacid sequences

The other difference copes with traceback algorithm. Maximum value of $M$ is considered as beginning cell, and alignment is carried out till a 0 is found in the matrix $M$. This will provide with the best local alignment, or accordingly to the previous nomenclature, $\tau$ will be aligned with $U$ with more score (less gaps) than with $V$.
However, and as it will be seen in Section II-C, performance of the biometric system will strongly rely on $\tau$ length, independently of the performance of the local alignment, since the lower the length of $\tau$, the lower the possibilities of recognizing a certain user. This fact is directly arisen from the nature of the template itself.

Finally, and as an example, Fig. 6 is presented to show two aligned templates, $T_{1}$ and $T_{2}$, belonging to the same user (above image), and to a different users (below image).
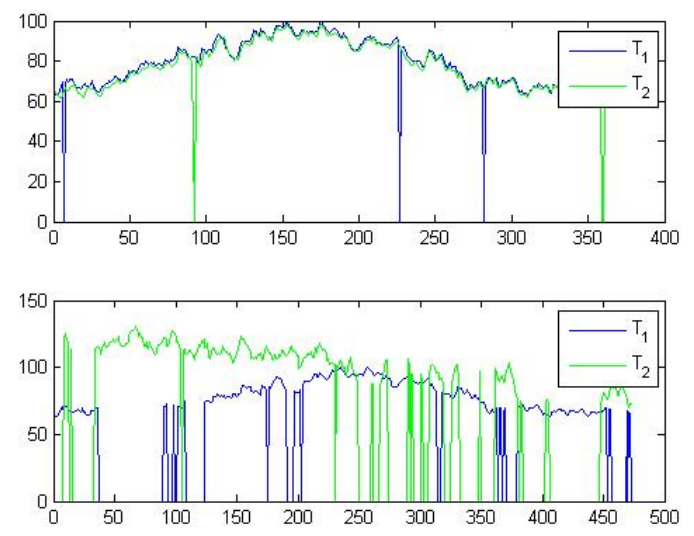

Fig. 6. Two comparisons: Above, two templates for the same user. Below, two templates for different users.

Note how the number of included zeros is a significative measure of similarity once the templates have been aligned.

\section{Results}

Several aspects of the algorithm are to be measured within this section. This algorithm has been tested with images contained in UPOL database, [5], which consists of 64 users with 3 images per eye, in other words, 384 images.

The results obtained here are compared to those in [8], in order to make a good comparison when using the same database.

Firstly, the whole biometric algorithm requires both irides for identification and authentication. Two scores are obtained, one score regards left eye, the other one regards right eye. However, only the minimum between them is considered as the final score, id est, that score to identify or authenticate a certain user.

Since there exist many different aspects to be measured, they will be separately detailed in subsequent subsections, for the sake of simplicity.

1) Temporal study: Regarding temporal aspects of the algorithm, it must be considered the fact that this alignment algorithm verifies to be a $O\left(n^{2}\right)$ algorithm, not only in time but also in memory, [4]. Obviously, temporal performance depends entirely on sequence length, being completely 
independent from $\sigma$ and $\mu$. Besides, all the experiments were taken place in a PC Intel Core 2 Duo @ $1.87 \mathrm{GHz}$, with 2GB Memory, and algorithms were implemented under MATLAB R2007.

Several performances of the global alignment algorithm pointed out that in average, aligning signals of 360 points in length, implies $\bar{t}=1.36 \mathrm{~s}$ and a standard deviation of $\sigma_{t}=0.01 \mathrm{~s}$. However, in order to measure both global and local alignment, Fig. 7 is provided, where several performances of local algorithm are carried out. Notice that a global alignment is a specific case of a local alignment when both templates contains the same number of elements.

Fig. 7 represents the variation in time when aligning two sequences but with different lengths. In $X$ and $Y$ axis, length of two templates are represented, while in Z-axis time is available in seconds. Maximum value is achieved for templates of length 720 , id est, two normal templates concatenated, only used as an example. Furthermore, Fig. 8 shows the exponential characteristic of the algorithm complexity.

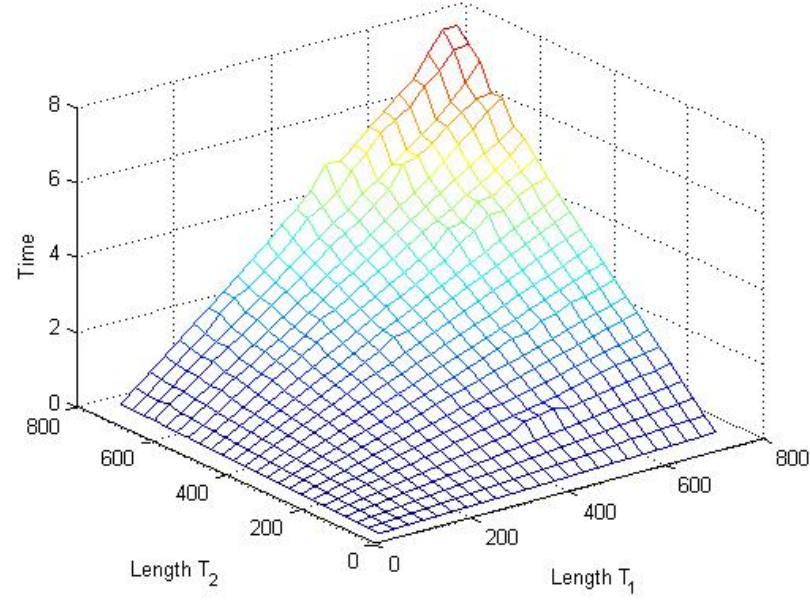

Fig. 7. Time variation in relation to templates lengths

2) Standard deviation study, $\sigma$ : In this subsection, how the performance of the systems varies in relation to $\sigma$ is studied. Never before in this paper has been suggested which is the key to manage the performance of the whole biometric system. Given a database, this responsibility relies completely on $\sigma$, the standard deviation of the gaussian distribution used to compared two elements in two templates, and on templates length which will be study in next subsection II-C3.

Fig. 9 represents the variation of EER in relation to $\sigma$. There exists an optimal value for $\sigma$ which minimizes EER. This value can be set by hand, or found out by using more sophisticated methods such as Evolutionary Strategies, [15]. However, this problem is beyond the scope of this paper and remains as future work.

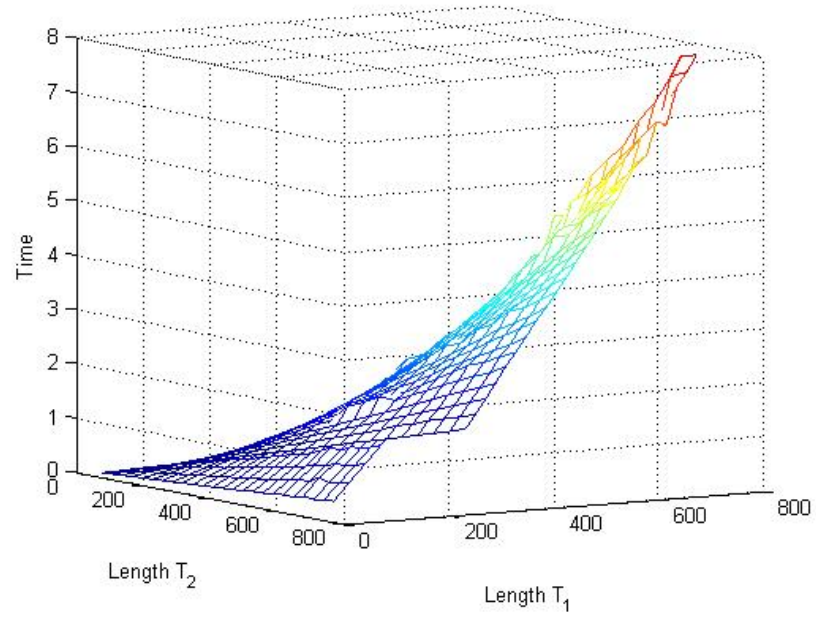

Fig. 8. Exponential aspect of the algorithm complexity

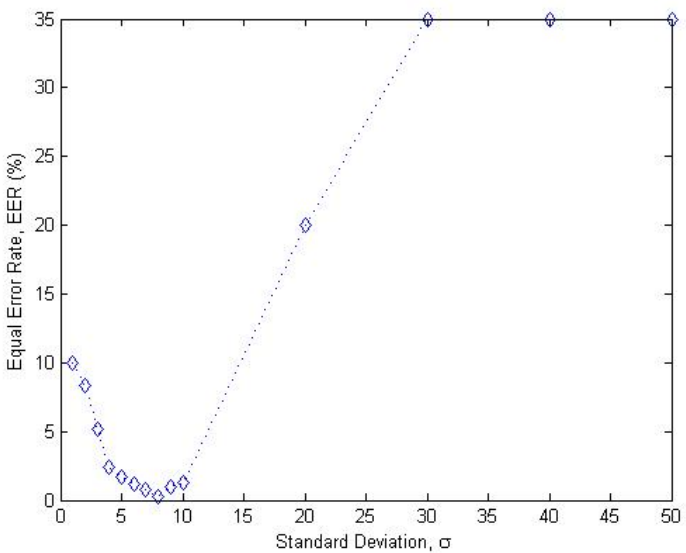

Fig. 9. Variation of EER due to standard deviation, $\sigma$

Notice the performance of the algorithm attains a value where is independent of $\sigma$. However, in those values, algorithm lacks of interest due to its low accuracy. Furthermore, $\sigma=8$ was selected to obtained best values.

3) Template length study: A proper study about the length of the templates is required, since it is obviously understandable the fact that the longer the templates, the better the performance could be. However, it remains to show to what extend this is true, or in other words, whether it could be possible to recognize a certain user by using a portion of a template.

In order to provide a form of measuring the previous query, Table I presents the relation between lengths of templates and EER, expressed in percentage (\%), and it was built by carrying out a normal comparison among users within the database, but taking different portions of each template. Such a portion was taken randomly from a different template from those used in the comparison, in an 
attempt to avoid the possibility of comparing a portion of a template and the same template where the portion was taken from, which is a trivial problem.

Table I provides an important result: even with short template lengths, identification or verification is possible with good accuracy considering the portion of template compared. Matrix diagonal is highlighted to remark the fact that maximum EER is achieved when lengths are the same in both templates. Notice Table I is symmetrical since $\zeta$ verifies so.

Furthermore, local alignment algorithm never fails in associating the respective portion to the corresponding template. However, avoiding completely the possibility of associating a portion to a not corresponding template, remains as future work.

\section{Performance}

Having described which parameters are related to EER, the performance of the whole biometric system is to be measured. In order to obtain results, which could be compared to those obtained in [8], similar experiments were carried out.

Correct Match Rate, CMR, was considered in [8], to express accuracy in a human iris recognition.

CMR equation is presented in (1),

$$
C M R=1-\left[F A R\left(\delta_{0}\right)+F R R\left(\delta_{0}\right)\right]
$$

where $F A R$ and FRR stand for False Acceptance Rate and False Rejection Rate, respectively, and $\delta_{0}$ represents the optimal threshold where CMR is maximum. Notice, $\delta$ in general represents the number of zeros in an alignment of two signals.

Table II compares the results achieved in [8], with the proposed method in this paper. Despite of not being provided in [8], EER is calculated for the alignment algorithm, with a result of $E E R=0.3 \%$. A remarkable result, although it cannot be compared to other EERs obtained with other algorithms, since databases are different. Rates in identifying and authenticating are $100 \%$ and $99.5 \%$, respectively.

TABLE II

COMPARISON OF RESULTS

\begin{tabular}{cccc}
\hline \hline Method & $F A R\left(\delta_{0}\right)[\%]$ & $F R R\left(\delta_{0}\right)[\%]$ & CMR [\%] \\
\hline Dobeš et al., [8] & 0.26 & 0.69 & 99.05 \\
Proposed & 0.3 & 0 & 99.7 \\
\hline \hline
\end{tabular}

On the other hand, better results are obtained, given the same database, with this new algorithm.

Regarding local alignment, accuracy evidently decrease when being performed, due to the length of the templates, as seen in Table I. However, local alignment is able to locate within a whole template where a portion of template belongs to, with awesome accuracy. On the other hand, a bigger template will be considered as future work in order to make local alignment achieve better results.

\section{CONCLUSIONS}

Aligning two Iris templates offers a new approach in a biometric system based on Iris detection. Results were compared in reference to those which were based on the same database, being clearly better. As an overall, $\mathrm{EER}=0.3 \%$ and $\mathrm{a} \mathrm{CMR}=99.7 \%$ were achieved. However, decreasing processing time remains as future work, as well as developing new metrics in order to measure similarities in this new alignment.

On the other hand, and considering these new algorithm characteristics, new scenarios arise where it can take place. First of all, this algorithm can be used as a normal procedure to identify or authenticate individuals. Secondly, it allows to identify a user with only a portion of template, there being a big template stored in the database, requiring from the user a partial template. This aspect decreases accessing time, and allows the system to recognize a certain user under low quality conditions such as partial occluded eyes, blurred images and so forth.

In addition to this, identifying an individual in a database requires a 1 : $N$ comparison. However, this new strategy allows to create a 'profile', [4], with all the individuals registered in the database, being possible to identify a certain user with a 1 : 1 comparison decreasing enormously the accessing time. This also remains as future work.

Furthermore, notice that when pupil dilates, Iris cells hide one over others, putting each on top of others depending to what extend the pupil dilates. If a new template is read without averaging in a radial direction, signals obtained by reading intensities along the radius, $\rho$, being $\alpha$ fixed, could be aligned among them, since deletion and insertion concepts correspond directly to those partial Iris cells hidden. In other words, when pupil dilates, this algorithm would remain invariant to such a situation. However, a deeper understanding of this phenomenon together with that new feature extraction are kept as future work, as well as an attempt to test this algorithm with other databases.

Finally, not only in Iris Detection Systems could this algorithm be used, but also on other biometric techniques. Reader should be aware of the fact that a DNA Alignment algorithm is one of the most powerful algorithms in comparing sequences, hence its promising results when being extended to a biometric context, and what is more, all the properties derived from this algorithm allow possibilities never before considered in Biometry.

\section{References}

[1] J. Daugman, 'How Iris Recognition Works', IEEE Transactions on Circuits and Systems For Video Technology, Vol. 14, n 1, January 2004. 
TABLE I

EER VERSUS TEMPLATE LENGTHS

\begin{tabular}{c|cccccccccc}
\hline \hline & 36 & 72 & 108 & 144 & 180 & 216 & 252 & 288 & 324 & 360 \\
\hline 36 & 14.29 & 27.2 & 28.34 & 26.03 & 27.78 & 28.37 & 30.27 & 30.8 & 31.32 & 32.59 \\
\hline 72 & 27.2 & 3.89 & 16.52 & 16.07 & 17.33 & 19.1 & 18.86 & 19.77 & 21.4 & 20.26 \\
\hline 108 & 28.34 & 16.52 & 2.55 & 9.3 & 11.91 & 10.61 & 11.35 & 11.46 & 10.92 & 11.9 \\
\hline 144 & 26.03 & 16.07 & 9.3 & 1.65 & 14.29 & 12.05 & 12.3 & 11.76 & 12.18 & 11.61 \\
\hline 180 & 27.78 & 17.33 & 11.91 & 14.29 & 1.1 & 7.14 & 7.14 & 7.14 & 7.14 & 7.14 \\
\hline 216 & 28.37 & 19.1 & 10.61 & 12.05 & 7.14 & 1.02 & 7.14 & 7.14 & 7.14 & 7.14 \\
\hline 252 & 30.27 & 18.86 & 11.35 & 12.3 & 7.14 & 7.14 & 0.6 & 3.29 & 1.65 & 1.65 \\
\hline 288 & 30.8 & 19.77 & 11.46 & 11.76 & 7.14 & 7.14 & 3.29 & 0.55 & 2.04 & 1.53 \\
\hline 324 & 31.32 & 21.4 & 10.92 & 12.18 & 7.14 & 7.14 & 1.65 & 2.04 & 0.41 & 1.09 \\
\hline 360 & 32.59 & 20.26 & 11.9 & 11.61 & 7.14 & 7.14 & 1.65 & 1.53 & 1.09 & 0.3 \\
\hline \hline
\end{tabular}

[2] A. de Santos Sierra, C. Sánchez Ávila, R. Sánchez Reíllo, Sistema de identificación biométrica mediante patrón de iris utilizando operadores morfológicos y representación, Congreso Iberoamericano de Seguridad Informática (CIBSI2007), November 2007, pp. 427-434, Mar del Plata, Argentina.

[3] R. P. Wildes. Iris recognition: an emerging biometric technology. In Proceedings of the IEEE, vol. 85, no.9, pp. 1348-1363, U.S.A., September 1997.

[4] R. Durbin, S. Eddy, A. Krogh, G. Mitchison, Biological sequence analysis, Cambridge University Press, UK, 1998.

[5] M. Dobeš and L. Machala. UPOL iris image database, 2004. http://phoenix.inf.upol.cz/iris/

[6] Y. Zhu, T. Tan, Y. Wnag, Biometric Personal Identification Based on Iris Patterns, Pattern Recognition, 15th International Conference on , Vol. 2 , pp. 801-804, 2000. Proceedings of SPIE 5612 15-11 Presented at SPIE Security and Defence, London, UK 2004.

[7] Y. Du, R. W. Ives, D. M. Etter, T. B. Welch, C. I Chang, A One Dimensional Approach to Iris Recognition, Proceedings of SPIE Volume 5404, Biometric Technology for Human Identification, pp. 237-247, April, 2004.

[8] M. Dobeš, L. Machala, P. Tichavský, J. Pospíšil Human eye iris recognition using the mutual information, Elsevier, Optik 115, No. 9 (2004), pp. 399-404.

[9] W. W. Boles, B. Boashash, A Human Identification Technique Using Images of the Iris and Wavelet Transform, Signal Processing, IEEE Transactions on, Vol. 46, No. 4, 1998.

[10] C. Sánchez-Ávila, R. Sánchez-Reíllo, D. de Martín-Roche, Iris-Based Biometric Recognition using Dyadic Wavelet Transform, Aerospace and Electronic Systems Magazine, IEEE, vol. 17, pp. 3-6, October 2002.

[11] S. N. Yanushkevich, Image pattern recognition : synthesis and analysis in biometrics, ed. World Scientific, May, 2007.

[12] S. B. Needleman, C. D. Wunsch, A general method applicable to the search of similarities in the amino acid sequence of two proteins. Journal of Molecular Biology 48:443-453, 1970.

[13] J. Mandel, The statisticial analysis of experimental data, Ed. Dover Publications, INC., New York, 1984.

[14] T. F. Smith, M. S. Waterman, Identification of common molecular subsequences. Journal of Molecular Biology.147:195-197, 1981.

[15] A. E. Eiben and J. E. Smith, Introduction to Evolutionary Computing, Berlin, Germany: Springer, 2003.

\section{VITA}

Alberto de Santos Sierra ad gradum promotus fuit in Escuela Técnica Superior de Telecomunicaciones (ETSIT) Matritense. Ad exitum studios duxit in Vrije Universiteit Amsterdam, in Hollandia, lulio mense, anno Domini MMVII. A mense Septembre laborat Centro Domótica Integral, CeDInt, Universitatis Polithecnicæ Matritensis. Biometria Stresæ, Systematis Reagnitionis Biometricæ et Cryp- tographia eius investigationis materia est. Nunc doctoris laurea inaugurandus est in ETSIT.

$\mathrm{He}$ received the degree of Telecommunication Engineer in Escuela Técnica Superior de Telecomunicaciones (ETSIT), finishing his studies in Vrije Universiteit Amsterdam with a Final Master Project based on Iris Recognition, in July, 2007. At present, he researches in Centro de Domótica Integral, CeDInt, in topics related to Biometric Recognition Systems, Stress Biometry and Cryptography. He is currently a PhD Student in ETSIT.

Carmen Sánchez Ávila received the PhD degree in Mathematical Sciences from the Polytechnic University of Madrid, Spain, in 1993. Since 1985 she has been with the Department of Applied Mathematics, Polytechnic University of Madrid, where she conducts research on Digital Signal Processing, Cryptography and Biometric.

At present she is Professor in the above mentioned Department where she has been teaching different undergraduate courses related to Mathematical and Numerical Analysis as well as graduate courses in Wavelets in Signal Processing and Biometrics Systems. Her interests in RD are still Cryptography, Biometrics and Security Systems.

Vicente Jara Vera obtained the degree of Telecommunication Engineer, specialized in Telematics, in Escuela Técnica Superior de Ingenieros de Telecomunicación (ETSIT), from Universidad Politécnica de Madrid (UPM) in 2003 with a Final Master Project based on Public Key Cryptography.

Professionally, he was a grant holder in Departamento de Ingeniería Telemática, (DIT-ETSIT), Systems Engineer and Programmer in Art2000 Group, Net Engineer in Net2S, Net Project Manager in HP and Net Consultant and Project Manager in Ámbar Telecomunicaciones, being nowadays a Researcher in Cryptography and Security by Department of Applied Mathematics (MAT-ETSIT) in Madrid and in Grupo de Biometría y Tratamiento Numérico de la Información. 